\title{
Histogram equalization and noise removal process for enhancement of image
}

\author{
Badri Narayan Mohapatra ${ }^{1 *}$ and Prangya Prava Panda ${ }^{2}$ \\ Assistant Professor, Department of Electronics \& Telecom Engineering, Oxford College of Engineering and \\ Management (OCEM), Bhubaneswar, Odisha, India ${ }^{1}$ \\ B.Tech Student, Department of Computer Science, The Techno School, Bhubaneswar, Odisha, India ${ }^{2}$
}

(C2017 ACCENTS

\begin{abstract}
For improving contrast the common methods is used in digital images is histogram equalization (HE).The output image look better when there is changing the pixels intensity while image enhancement process going on. Improve an image sometimes defined by modifying the intensities or colors or increasing the SNR value. Different filtering as well as enhancement method makes image analysis and processing to achieving a satisfactory level. Performance degrades only when there will be noise in the image. So in this paper we analyses progressive switching median filer (PSMF) and decision-based algorithm (DBA) techniques for image enhancement. For supressing efficient denoising scheme DBA and PSMF is utilized here. Test results for PSMF and DBA makes good efficiency in removing salt and pepper noise for different noise density level.
\end{abstract}

\section{Keywords}

Histogram, Image enhancement, Denoising, PSMF.

\section{Introduction}

Image (black-and-white) denoted $\mathrm{f}(\mathrm{x}, \mathrm{y})$ for twodimensional image. Color images are three twodimenisional arrays. Color images are referred to as red, green, and blue i.e RGB. The (intensity or brightness) histogram shows how many times a particular grey level (intensity) appears in an image. For example, 0 as black, 255 as white. An image has low contrast when the complete range of possible values is not used. Histogram of color images. RGB color can be converted to a gray scale value by $\mathrm{Y}=$ $0.299 \mathrm{R}+0.587 \mathrm{G}+0.114 \mathrm{~B} \mathrm{Y}$ : the gray scale component in the YIQ color space used in NTSC television [1]. The weights reflect the eye's brightness sensitivity to the color primaries.

$S_{k}=T\left(r_{k}\right)=\sum_{j=0}^{k} \frac{n_{j}}{n}(L-1)$

$\mathrm{k}=0,1,2, \ldots, \mathrm{L}-1$

L: number of grey levelsin image (e.g., 255)

$\mathrm{n}_{\mathrm{j}}$ : number of times $\mathrm{j}$-th grey level appears in image $\mathrm{n}$ : total number of pixels in the image.

\section{Histogram processing}

Histogram is the discrete function $h(r k)=n k$, where $\mathrm{rk}$ is the kth gray level in the range of $[0, \mathrm{~L}-1]$ and $\mathrm{nk}$ is the number of pixels having gray level rk[2].

*Author for correspondence

22
Histogram equalization is a method which increases the dynamic range of the gray-levels in a lowcontrast image to cover full range of gray-levels [3].

Histogram equalization:the new intensity value of pixel $\mathrm{x}$ is calculated by

$I(x)=\operatorname{round}\left[\frac{c d f(x)-\min c d f}{1-\min c d f}\right] \times(L-1)$

Histogram equalization : the probability function of the output levels is uniform. The transformation function is simply the CDF.

Histogram equalization can be used to improve the visual appearance of an image.

Calculate the cumulative distribution function (CDF) of the image using formula:

$\operatorname{CDF}[\mathrm{k}]=\sum_{\mathrm{j}=0}^{\mathrm{k}} 255^{\mathrm{n}_{\mathrm{j}}} / \mathrm{N}$

Where CDF[k] is the CDF value of pixel with value $\mathrm{k}$ in input image, is the number of pixels with value $j$ and $\mathrm{N}$ is the total number of pixels.

Re-label all input pixels with corresponding values $\mathrm{CDF}[\mathrm{k}]$.

HE is most extensively utilized contrast enhancement technique due to its simplicity and ease of 
implementation. Applications of histogram equalization are found in many areas such as medical image processing, texture synthesis, as well as speech recognition [4]. Image contrast enhancement also can be done using Exposure based Sub Image Histogram [5]. Contrast image enhancement can be done by histogram modification framework [6]. Because of high efficiency and high quality HE (Histogram Equalization) has achieved most attention among other methods in image enhancement [7]. A strong technique $\mathrm{HE}$ is used for improving contrast in images also image histogram provides a description about image attributes and its appearance [8].

Noise within image due to enrolment, preparation also in image acquisition process so superior restoration methods needed to suppression of saltand-pepper noise. Due to its efficient computational performance and superiority median filter is considered. Filtering scheme based on the iterative applications can possible to remove noisy images [9]. From the corrupted image switching-based adaptive weighted mean filter will help to remove salt-andpepper noise [10]. Trimmed Median filter (decision based) can remove high density salt and pepper noise [11]. For removal of Salt and Pepper noise from images decision based algorithm performance is excellent [12] and [13]. Figure 1 shows the original and histogram image. Figure 2 shows the histogram equalization of moon TIF image.
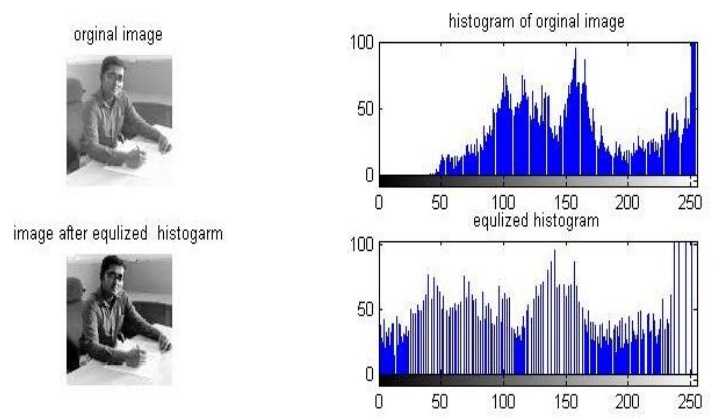

Figure 1 Original own image histogram

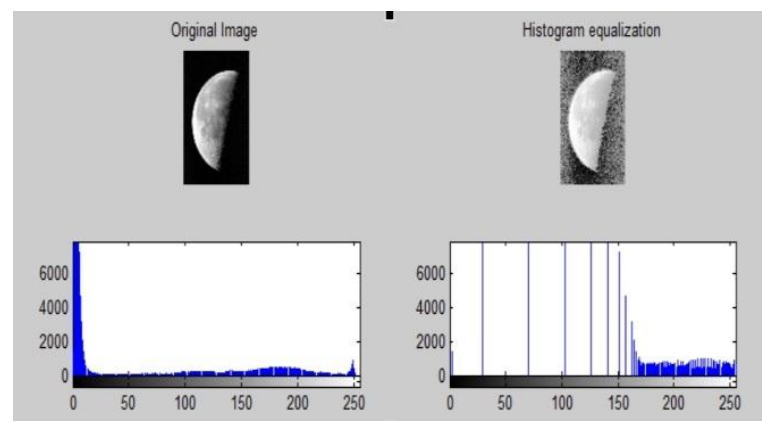

Figure 2 Histogram equalization of moon TIF image 23
Figure 3 shows the histogram equalization of Pout.tif image.

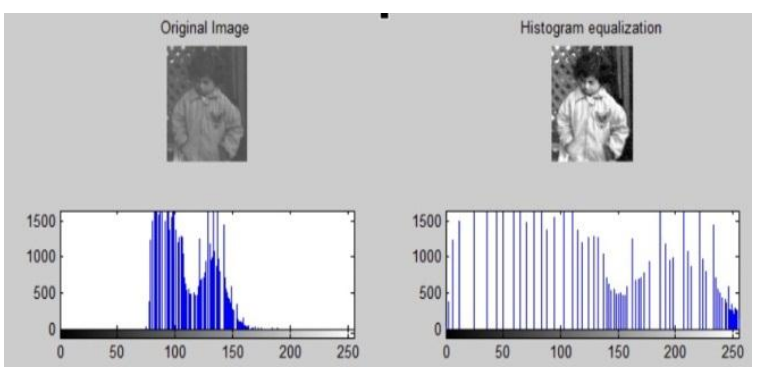

Figure 3 Histogram equalization of Pout.tif image

\section{Filtering scheme}

In image processing Noise reduction is a Challenging problem. In communication channel due to error images are corrupted. Before some subsequent processing noise elimination from noise should be require. Various filters designed for noise removal in digital images acquisition and transmission process. Progressive switching median filter works as impulse detection and in the next stage next stage noise filtering. PSMF and DBA based algorithm will help to remove those errors from corrupted images. The above two filters are tested with salt and pepper Image. For detecting and removing salt and pepper noise non-linear median filter PSMF is used which perform in spatial. If we compare standard median filter, Adaptive median filter PSMF is better filter efficiency. Figures 4 and 5 shows the PSMF and DBA results respectively.

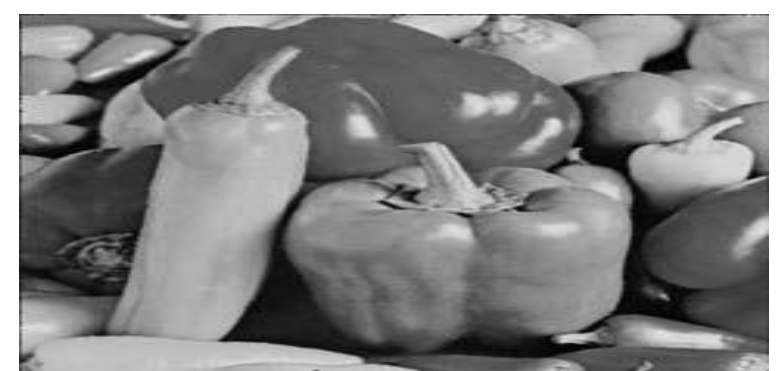

Figure 4 PSMF result

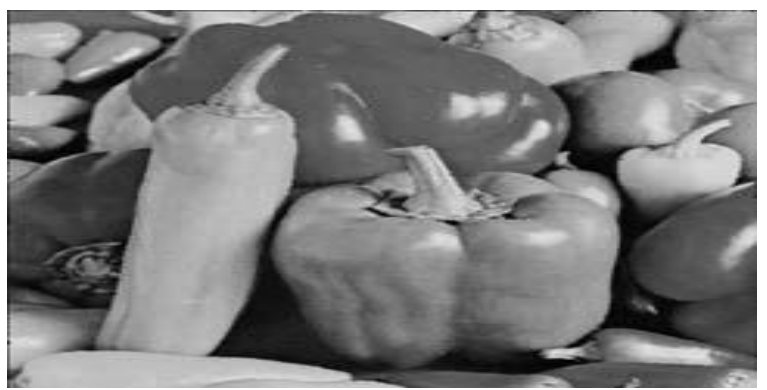

Figure 5 DBA result 
Figure 6 shows the original salt pepper image. Table 1 shows the comparison of the results.

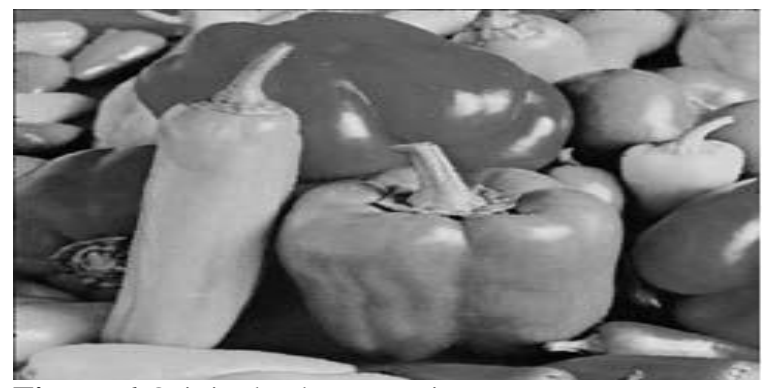

Figure 6 Original salt pepper image

Table 1 Comparison chart

\begin{tabular}{llll}
\hline S. No & $\begin{array}{l}\text { Noise } \\
\text { density }\end{array}$ & $\begin{array}{l}\text { Decision } \\
\text { based } \\
\text { algorithm }\end{array}$ & $\begin{array}{l}\text { Progressive } \\
\text { switching } \\
\text { median } \\
\text { filter }\end{array}$ \\
\hline 1 & 20 & 34.71 & 31.21 \\
2 & 40 & 30.95 & 28.56 \\
3 & 60 & 27.52 & 25.73 \\
4 & 80 & 23.47 & 14.01 \\
\hline
\end{tabular}

All the figures of results are calculated in Matlab. A fixed $3 \times 3$ window is used for filtering in DBA. Figure 7 shows the comparison result of PSMF and DBA in terms of PSNR values with respect to Noise density (ND \%).

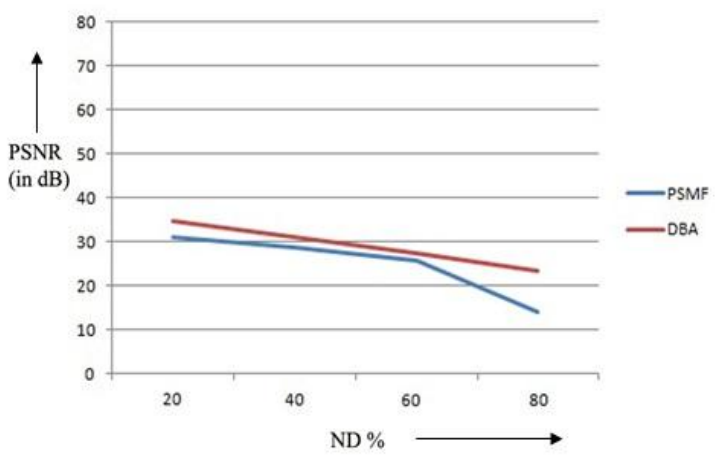

Figure 7 The visual graph presented for the purpose of outperforms of DBA and PSMF

\section{Conclusion and future work}

In this paper HE of different images like own image as well as moon and pout.tif image in Matlab has been computed. Also for removing salt pepper noise a comparative results have been discussed. Between DBA and PSMF a quantitative comparison based on PSNR is performed. Noise density levels for peppersbmp range at various percentages as 20, 40, 60 and 80 are calculated. PSMF will take more computing time compared to DBA. De-noising scheme DBA 24 and PSMF is introduced for suppressing salt and pepper noise. When the range is computed then the above denoising scheme method will be able to perform good result. Noise blotches can also be detected and filtered by PSMF. Median filter process only noise pixels by DBA. Further research will find image enhancement factor (IEF) as well as removal of (corrupted pixels) high density salt and pepper noise.

\section{Acknowledgment}

None.

\section{Conflicts of interest}

The authors have no conflicts of interest to declare.

\section{References}

[1] Plataniotis KN, Venetsanopoulos AN. Color image processing and applications. Springer Science \& Business Media; 2013.

[2] Haller EA. Adaptive histogram equalization in GIS. Annals of the University of Craiova-Mathematics and Computer Science Series. 2011; 38(1):100-4.

[3] Celik T, Tjahjadi T. Automatic image equalization and contrast enhancement using Gaussian mixture modeling. IEEE Transactions on Image Processing. 2012; 21(1):145-56.

[4] Wang Q, Ward RK. Fast image/video contrast enhancement based on weighted thresholded histogram equalization. IEEE Transactions on Consumer Electronics. 2007; 53(2):757-64.

[5] Chen SD. A new image quality measure for assessment of histogram equalization-based contrast enhancement techniques. Digital Signal Processing. 2012; 22(4):640-7.

[6] Arici T, Dikbas S, Altunbasak Y. A histogram modification framework and its application for image contrast enhancement. IEEE Transactions on Image Processing. 2009; 18(9):1921-35.

[7] Wu X, Liu X, Hiramatsu K, Kashino K. Contrastaccumulated histogram equalization for image enhancement. 2017.

[8] Singh RP, Dixit M. Histogram equalization: a strong technique for image enhancement. International Journal of Signal Processing, Image Processing and Pattern Recognition. 2015; 8(8):345-52.

[9] Ghanekar U, Singh AK, Pandey R. A contrast enhancement-based filter for removal of random valued impulse noise. IEEE Signal Processing Letters. 2010; 17(1):47-50.

[10]Zhang X, Xiong Y. Impulse noise removal using directional difference based noise detector and adaptive weighted mean filter. IEEE Signal Processing Letters. 2009; 16(4):295-8.

[11] Esakkirajan S, Veerakumar T, Subramanyam AN, PremChand $\mathrm{CH}$. Removal of high density salt and pepper noise through modified decision based 
unsymmetric trimmed median filter. IEEE Signal Processing Letters. 2011; 18(5):287-90.

[12] Kumar RR, Vasanth K, Rajesh V. Performance of the decision based algorithm for the removal of unequal probability salt and pepper noise in images. In international conference on circuit, power and computing technologies 2014 (pp. 1360-5). IEEE.

[13] Kishorebabu V, Packyanathan G, Kamatham H, Shankar V. An adaptive decision based interpolation scheme for the removal of high density salt and pepper noise in images. EURASIP Journal on Image and Video Processing. 2017; 2017(1):67.

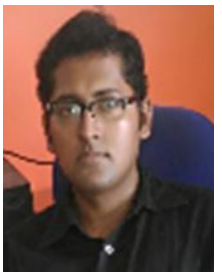

Badri Narayan Mohapatra started his research on communication in Centurian University, Odisha. He did his B.E. from Berhempur University and M.Tech from Biju Pattnaik University of Technology. His research area covers Light propagation, Digital Signal and Image Processing.

Email: badri1.mohapatra@gmail.com

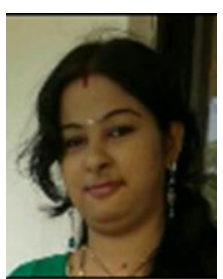

Prangya Prava Panda did her Diploma from Government Polytechnic, Bhubaneswar, Odisha. Now she is continuing B.Tech in CSE Department in The Techno School, Bhubaneswar. 